\title{
Analysis on Optical System Performance of A Strap-down Uncooled Infrared Detector
}

\author{
Zhan Zhang ${ }^{1, \mathrm{a}}$, Guanglin $\mathrm{He}^{1, \mathrm{~b}}$ and Pan Xiao ${ }^{1, \mathrm{c}}$ \\ ${ }^{1}$ Beijing Institute of Technology - School of Mechatronical Engineering, Beijing 100081, China. \\ apeakcanghai@live.com, bhe8507@aliyun.com, casas0601@163.com
}

Keywords: guided munition; optical system; performance index; resolution

\begin{abstract}
According to the requirements of micro guided munition on detection, a design method on optical system indexes of the strap-down uncooled infrared detector is put forward. Based on the structure, mathematical modeling of the optical system is completed, and a series of basic parameters are calculated and demonstrated. Finally, the detection model is built by considering variation of trajectory parameters, from which getting the resolutions of typical targets. The results show that all resolutions simulated can fit the minimum when the detector working in different conditions, and can certainly meet the tactical guidelines.
\end{abstract}

\section{Introduction}

In order to meet the needs of the war in the future, using of infrared detection technology in the military is moving towards high sensitivity, wide spectrum, high resolution, low power consumption, miniaturization and intelligent [1].

The Polish, Rogalski, analyzed the current demand for the development of infrared detector, and proposed the 3 parts included in the third generation infrared detector as follows [2]:

(1) high performance, high resolution cooled imagers having multi-color bands,

(2) medium- to high-performance uncooled imagers,

(3) very low cost, expendable uncooled imagers.

The relationship between 3 items mentioned above is "or", which means as long as a detector meets one of the requirements can be called the third generation infrared detector [3].

The infrared detector has advantages of low power consumption, light weight, small volume, high starting and response speed etc. [4]. It has been widely used in large-scale military infrared equipment. Many achievements have been made in the design of the optical system of the infrared detector, and some products have been applied in the project $[5,6,7]$. Most of the current designs are based on given performance parameters, whose design and demonstration are lacking. In this paper, a design method of the performance parameters of the optical system of a strap-down infrared detector is presented, and is verified by simulation detection of typical targets.

\section{Main parameters design of the optical system}

\subsection{Design requirements}

The detector is the important part to find the target and form a stable and clear image, measure the offset parameters between true trajectory and the ideal, and then generate control instructions [8]. Components of strap-down uncooled infrared detector as shown in Fig. 1:

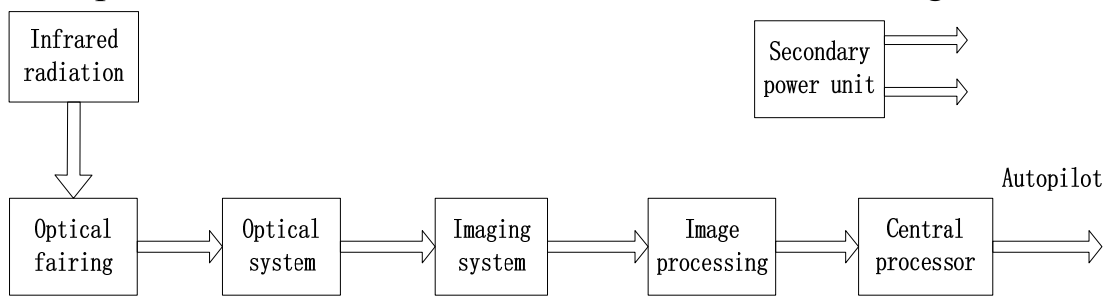

Fig. 1 Strap-down uncooled infrared detector

The performance of the optical system has a direct impact on the performance of whole detector, 
and quite a number of technical indicators must be implemented by the optical system, such as the instantaneous field of view distance and blind area.

\subsection{Focal length}

The focal length of the optical system is related to the field of view, and the relationship between them is as follows:

$$
f \leq D_{0} / 2 \tan \frac{\omega}{2}
$$

In formula (1), $f$ is the focal length, $\omega$ is the angle of field, and $D_{0}$ is the aperture, which can be designed by the following formula:

$$
D_{0} \geq 2.44 \frac{\lambda_{\max }}{\theta_{T}} N_{g}
$$

In formula (2), $\lambda_{\max }$ is the maximum wavelength required by the optical system, $\theta_{T}$ is the spatial resolution of targets required by the system, $N_{g}$ is the diffraction limited diffusion coefficient, and is set of 1.5 in design.

It can be seen from (1) and (2) that the focal length and field design is a crossed progress, and is restricted by the structure of system. In order to determine the range of focal length preliminary, design could be pushed on basis of parameters such as detection distance and optical lens specifications. The distance requirement of the detector is $1000 \mathrm{~m}$ or less. The optical lens specifications commonly used are 1/2 inch, 1/3 inch and 1/4 inches, and the parameters are given in Table 1

Table 1 Lens parameters

\begin{tabular}{cccc}
\hline Size (inch) & $1 / 2$ & $1 / 3$ & $1 / 4$ \\
\hline Width $(\mathrm{mm})$ & 6.4 & 4.8 & 3.6 \\
Length $(\mathrm{mm})$ & 4.8 & 3.6 & 2.4
\end{tabular}

The focal length can be calculated by the following formulas:

$$
\begin{aligned}
& f=u \times(D / U) \\
& f=h \times(D / H)
\end{aligned}
$$

In (3) and (4), is the actual width of the object, is the actual height of the object, is the distance between lens and object, is the image width, and is the image height.

When the target size is $3 \mathrm{~m} \times 3 \mathrm{~m}$, then it can be identified only if it covers an area over $1 / 16$ of the whole image. Focal lengths of different lens under different detection ranges, as shown in Table 2.

\subsection{Field of view}

\begin{tabular}{cccc} 
Table 2 Focal lengths in different distances (mm) \\
\hline Listance $(\mathrm{m})$ & $1 / 2$ & $1 / 3$ & $1 / 4$ \\
\hline 1000 & & & \\
500 & 80 & 106.7 & 80 \\
300 & 48 & 32 & 24 \\
100 & 10 & 7.5 & 5 \\
\hline
\end{tabular}

The angle of field is usually used to characterize the scope of observation. The angle means all objects in the field are under the range of imageable distances. Formulas of the angle of field is as follows:

$$
\begin{aligned}
& \omega_{u}=2 \operatorname{tg}^{-1}\left(\frac{u}{2 f}\right) \\
& \omega_{h}=2 \operatorname{tg}^{-1}\left(\frac{h}{2 f}\right)
\end{aligned}
$$

$\omega_{u}$ is the horizontal viewing angle, and the $\omega_{h}$ means vertical viewing angle.

In order to ensure all lenses of different specifications have a large field of view, several typical 
values of focal length within $10 \sim 50 \mathrm{~mm}$ are taken to calculate the angle of field. Results are included in Table 3 and Table 4

Table 3 Horizontal viewing angle $\left(\omega_{u}\right)$

\begin{tabular}{ccccccc}
\hline$f(\mathrm{~mm})$ & 10 & 15 & 24 & 32 & 40 & 50 \\
Lens size(inch) & & & & & & \\
\hline $1 / 2$ & 35. & 24. & 15. & 11. & 9.1 & 7.3 \\
$1 / 3$ & 27 & 18. & 11. & 8.6 & 6.9 & 5.5 \\
$1 / 4$ & 18. & 12. & 7.6 & 5.7 & 4.6 & 3.7 \\
\hline Table 4 Vertical viewing angle $\left(\omega_{h}\right)$ & & \\
\hline$f(\mathrm{~mm})$ & 10 & 15 & 24 & 32 & 40 & 50 \\
Lens size(inch) & & & & & & \\
\hline $1 / 2$ & 27 & 18. & 11. & 8.6 & 6.9 & 5.5 \\
$1 / 3$ & 20. & 13. & 8.6 & 6.4 & 5.1 & 4.1 \\
$1 / 4$ & 13. & 9.1 & 5.7 & 4.3 & 3.4 & 2.7 \\
\hline
\end{tabular}

\subsection{Blind area of detection}

The blind area of detection can be divided into region beyond the field and region within the field but cannot be recognized. The range of the former is $360^{\circ}-\omega_{u}$ and $360^{\circ}-\omega_{h}$, while it comes to critical point of the latter when the field is filled by the target image.

Following is the calculation of the critical point: Diameter is the longest string of a circle, so the distance will come to the maximum when the optical axis is perpendicular to the target. Formula is as follows:

$$
d=\frac{H}{2 \operatorname{tg} \frac{\omega_{h}}{2}}
$$

In this formula, is the distance in critical point. When, effective identification can be achieved. Taking $f=10 \mathrm{~mm}$ and $f=50 \mathrm{~mm}$ as the initial conditions, and the critical condition is the target image covers $1 / 16$ part of the field, precise critical points are shown in table 5 and table 6.

\begin{tabular}{cccc}
\multicolumn{4}{c}{ Table 5 critical value of blind area $(f=10 \mathrm{~mm})$} \\
\hline Lens size (inch) & $1 / 2$ & $1 / 3$ & $1 / 4$ \\
\hline Distance (m) & 100 & 133.3 & 200 \\
Critical value(m) & 6.25 & 8.3 & 12.5 \\
\hline \multicolumn{4}{l}{ Table 6 critical value of blind area $(f=50 \mathrm{~mm})$} \\
\hline Lens size (inch) & $1 / 2$ & $1 / 3$ & $1 / 4$ \\
\hline Distance (m) & 250 & 333.3 & 500 \\
Critical value $(\mathrm{m})$ & 31.25 & 41.9 & 63.7
\end{tabular}

From tables above, a conclusion can be drawn, regardless to the specifications of lenses, that critical values of blind area within the field are entirely less than $65 \mathrm{~m}$, a value which is quite ideal.

\subsection{Final determination of main parameters}

Considering the structure and cost of guidance system, a 1/4 inch lens is chosen. For getting higher image resolution and greater applicability, use resolution of $384 \times 288$ to replace $320 \times 240$ [9]. Relying on the structure as well formula (1), (2), and (7), the accurate parameters of the optical system are raised and optimized as follows.

Detection range: about $1 \mathrm{~km}$ (Atmospheric visibility $>10 \mathrm{~km}$, difference in temperature between target and background $>6 \mathrm{~K}$, and for $3 \mathrm{~m} \times 3 \mathrm{~m} \times 5 \mathrm{~m}$ target front cover $3 \times 3$ pixels). Angle of field: $9.6^{\circ} \times 7.2^{\circ}$. Focal length: $57 \mathrm{~mm}$. Pixel: $25 \mu \mathrm{m} \times 25 \mu \mathrm{m}$. Wavelength range: $8 \sim 14 \mu \mathrm{m}$. 


\section{Resolution analysis}

The resolution of the detector is an important factor that affects the target recognition. Resolution is a function of the distance, the angle of field, the obliquity of trajectory and the angle of attack. It can be calculated as follows

$$
\begin{aligned}
& x=\{l / L \cos \theta[\tan (\theta+\beta / 2 \pm \alpha)-\tan (\theta-\beta / 2 \mathrm{~m} \alpha)]\} \cdot X \\
& y=\{w / L \cos \theta[\tan (\theta+\gamma / 2 \pm \alpha)-\tan (\theta-\gamma / 2 \mathrm{~m} \alpha)]\} \cdot Y
\end{aligned}
$$

$x$ is the pixel in length direction, $l$ is the actual length of the target, $w$ is the actual width of the target, $L$ is the distance between the missile and the target, $\theta$ is the obliquity of trajectory, $\beta$ is the angle of field in length direction, $\gamma$ is the angle of field in width direction, $\alpha$ is the angle of attack, $X$ is the pixel of the sensor in length direction, and $Y$ is the pixel of the sensor in length direction. The geometric model of the detector in working mode is given in Fig. 2.

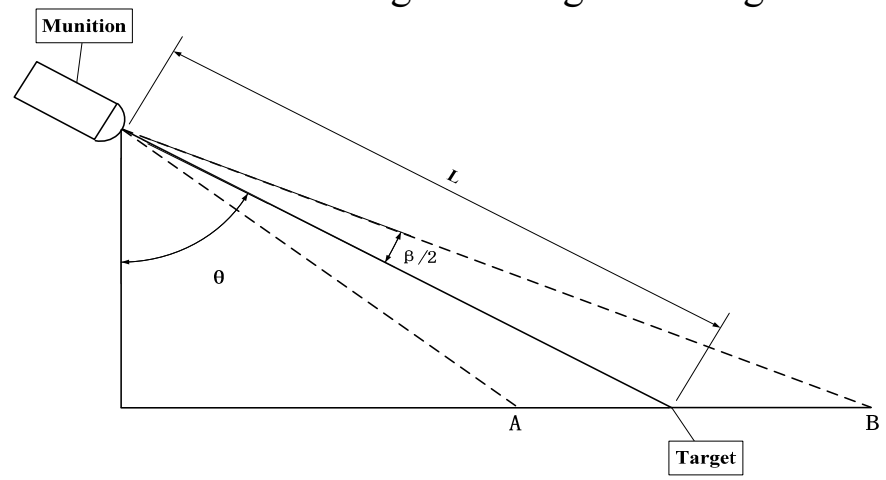

Fig. 2 Diagram of working mode of the detector

Using parameters of the detector in formula (8) and (9), the recognition ability was obtained in variable attack angles and obliquities of trajectory under circumstances of different distances. Table 7 shows the resolution of the detector to typical targets at $0^{\circ}$ angle of attack, and the obliquity of trajectory is variable.

Table 7 Resolution to typical targets $\left(\alpha=0^{\circ}\right)$

\begin{tabular}{cccc} 
Obliquity $(\mathrm{L}=600 \mathrm{~m})$ & $0^{\circ}$ & $50^{\circ}$ & $60^{\circ}$ \\
\hline M1A1 & $30.17 \times 13.94$ & $19.20 \times 8.91$ & $14.77 \times 6.88$ \\
Leopard 2 & $29.30 \times 14.11$ & $18.65 \times 9.02$ & $14.34 \times 6.97$ \\
T90 & $26.14 \times 12.86$ & $16.64 \times 8.22$ & $12.79 \times 6.35$ \\
M109 & $25.84 \times 12.01$ & $16.44 \times 7.68$ & $12.65 \times 5.94$ \\
Pzh2000 & $31.90 \times 13.66$ & $20.30 \times 8.73$ & $15.61 \times 6.75$ \\
2S3 & $29.72 \times 12.21$ & $18.91 \times 7.80$ & $14.55 \times 6.03$ \\
M992 & $25.84 \times 11.83$ & $16.44 \times 7.56$ & $12.65 \times 5.84$ \\
BTR-80 & $29.15 \times 11.06$ & $18.55 \times 7.07$ & $14.27 \times 5.47$ \\
\hline
\end{tabular}

Under the same distance, resolution decreases with the increase of obliquity of trajectory and angle of attack. Within the allowed range, the influence of attack angle on the resolution is less than that of the obliquity of trajectory. In condition of 60-degree obliquity of trajectory, resolution of the detector is still in $3 \times 3$ pixels above.

\section{Summary}

For a low-cost strap-down uncooled infrared detector, the field of view, the blind area of detection, the resolution and other key indexes can satisfy requirements of generalized guided munitions. With the development of image processing, visual tracking and etc., there will be a great promotion on performance of the detector, so the same in cost-effectiveness of munitions. 


\section{Reference}

[1] Zhang Xue, Liang Xiaogeng. Development needs of infrared detector. Electronics Optics \& Control. Vol. 20 (2013) No. 2, p. 41-45.

[2] Rogalski, A. New material systems for third generation infrared photodetectors. Opto-Electronics Review Vol. 16 (2008) No. 4, p.458-482.

[3] Shi Yanli. Choice and development of the third-generation infrared detectors. Infrared Technology. Vol. 35 (2013) No. 1, p. 1-8.

[4] Liu Ziji: Study on uncooled infrared focal plane detector testing and imaging technology (PhD, University Of Electronic Science And Technology Of China, China 2012). p. 12-14.

[5] Jang Chengzhou, Duan Meng, Pan Guoqing et al. A miniaturized optical system design of seeker with detector fixation. Infrared Technology. Vol. 36 (2014) No. 9, p. 743-747.

[6] Xiang Jiansheng, Pan Guoqing, Zhang Yunqiang. The design of catadioptric and reimaged LWIRA thermalization system. Infrared Technology. Vol. 34 (2012) No. 11, p. 644-647.

[7] Zhang Xin, Jia Hongguang. $90^{\circ}$ off-axis angle infrared optical system of roll-pitch seeker. Infrared and Laser Engineering. Vol. 42 (2013) No. 1, p. 143-147.

[8] Zhang Guangyi, Yang Jun, Zhu Xueping et al. Uncooled infrared imaging seeker. Northwestern Polytechnical University Press, 2009, p.7-10.

[9] Cang Lili: Research on the processing technology of uncooled infrared image based on $384 *$ 288 detector. (M.E, Nanjing University Of Science And Technology, China 2009). p. 2-5. 